\title{
GLOBAL LAND COVER CLASSIFICATION USING MODIS SURFACE REFLECTANCE PRODUCTS
}

\author{
Haruhisa Shimoda, Kiyonari Fukue \\ ${ }^{a}$ Tokai University Research and Information Center, 2-28-4 Tomigaya, Shibuya-ku, Tokyo 151, Japan - \\ (smd, fku)@keyaki.cc.u-tokai.ac.jp
}

\author{
Commission VIII, WG VIII/8
}

KEY WORDS: Global, Land Cover, Classification, Algorithms, Multi-temporal, Multi-spectral

\begin{abstract}
:
The objective of this study is to develop high accuracy land cover classification algorithm for Global scale by using multi-temporal MODIS land reflectance products. In this study, time-domain co-occurrence matrix was introduced as a classification feature which provides time-series signature of land covers. Further, the non-parametric minimum distance classifier was introduced for timedomain co-occurrence matrix, which performs multi-dimensional pattern matching for time-domain co-occurrence matrices of a classification target pixel and each classification classes. The global land cover classification experiments have been conducted by applying the proposed classification method using 46 multi-temporal(in one year) SR(Surface Reflectance 8-Day L3) and NBAR(Nadir BRDF-Adjusted Reflectance 16-Day L3) products, respectively. IGBP 17 land cover categories were used in our classification experiments. As the results, SR product and NBAR product showed similar classification accuracy of $99 \%$.
\end{abstract}

\section{INTRODUCTION}

Land cover maps of global or continental scale are basic information for many kinds of applications, i.e. global change research, modeling, resource management, etc. Several kinds of global land cover maps has been generated, such as IGBP DISCover Global Land Cover, UMD Global Land Cover, and MODIS Land Cover, etc., and these products have been distributed widely. However, accuracies of these global land maps were not sufficiently high. Most of these land cover maps were generated mainly using NDVI and its seasonal changes. However, NDVI data lose most of information contents which were originally included in many channels.

The objective of this study is to develop high accuracy land cover classification algorithm for global scale by using multitemporal MODIS land reflectance products. There are two kinds product of Surface Reflectance 8-Day L3 product and Nadir BRDF-Adjusted Reflectance 16-Day L3 product. Both are composed of 7 spectral bands $(620-670 \mathrm{~nm}, 841-876 \mathrm{~nm}$, 459-479nm, 545-565nm, 1230-1250nm, 1628-1652nm, and $2105-2155 \mathrm{~nm}$ ) with $500 \mathrm{~m}$ ground resolution. The former is the atmospheric corrected surface reflectance, while the latter corrects the BRDF effects in addition to the atmospheric correction. In this report, these products are called SR product and NBAR product, respectively.

\section{STUDY AREA AND SOURCE DATA SET}

The target area set in this study covers $140^{\circ}$ (from $70^{\circ}$ north to $-70^{\circ}$ south) and $360{ }^{\circ}$ for latitude and longitude direction, respectively. The region is covered by about 280 sinusoidal projection(SIN) grids which are distribution granule of SR and NBAR products (as shown in Figure 1). The SR and NBAR products of about 280 SIN grids were mosaicked and transformed to geographic longitude-latitude coordinate system with 0.005 degree interval as shown in Figure 2. This processing was performed by using MODIS Reprojection Tool
(MRT) which has been distributed from Land Processes DAAC. Because SR and NBAR products have been produced in eightday period, mosaic images of 46 scenes were generated as classification target data set for one year of 2007.

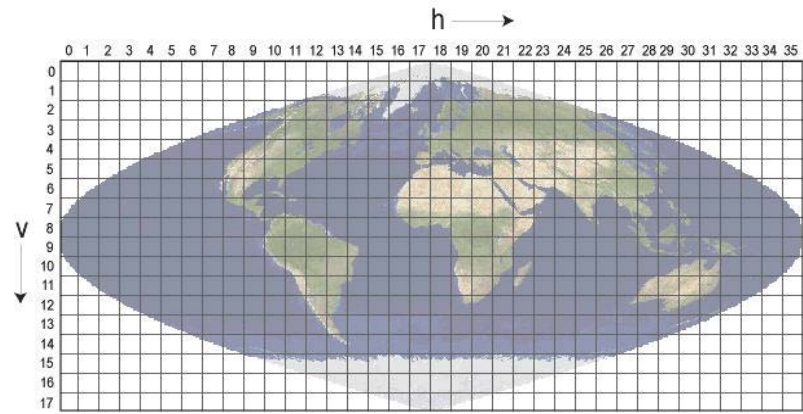

Figure 1. SIN grid.

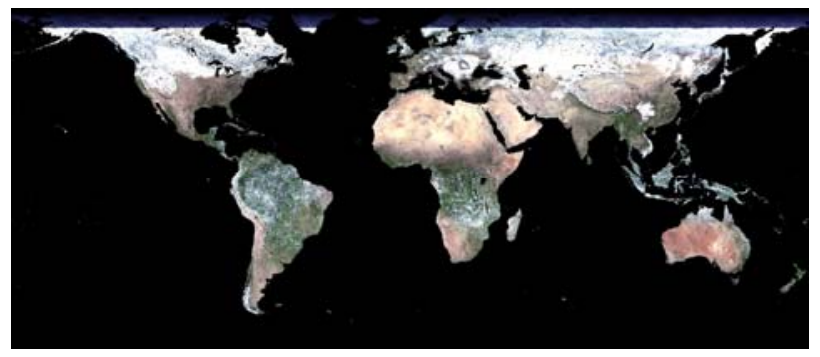

Figure 2. A result of mosaic and geometric transform. ( the scene of 2007.01.01)

\section{PROPOSED CLASSIFICATION ALGORITHM}

\subsection{Classification Feature}

In this study, time-domain co-occurrence matrix was introduced as a classification feature which provides time-series signature 
of land covers. Each elements (i, j) of the time-domain cooccurrence matrix is defined as probability that two pixels with a specified time-separation delta-t in the same spatial position have pixel value $i$ and $j$. Conventional co-occurrence matrix(that is spatial domain co-occurrence matrix) represents spatial texture while the proposed time-domain co-occurrence matrix represents time-series signature.

Figure 3a shows pixel values of annual time-series data conceptual. The time-domain co-occurrence matrices shown in Figure $3 \mathrm{~b}$ are derived from this time-series data in the case of one month separation. That is, a time-series changing pattern of pixel values produces the corresponding probability distribution pattern in the matrix. Time-domain co-occurrence matrix takes advantage of robustness against data loss and noise derived from cloud and undesirable fluctuation of calculated reflectance values.

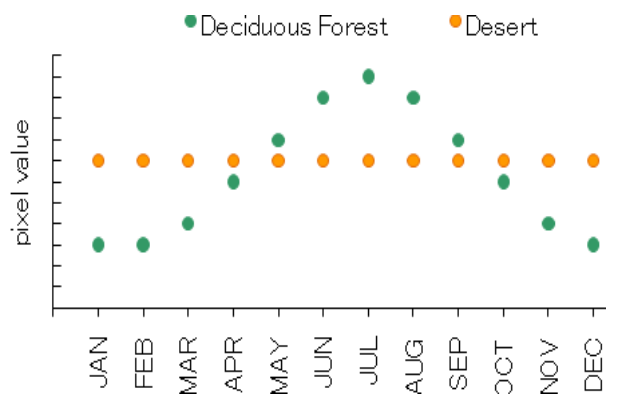

(a) annual time-series
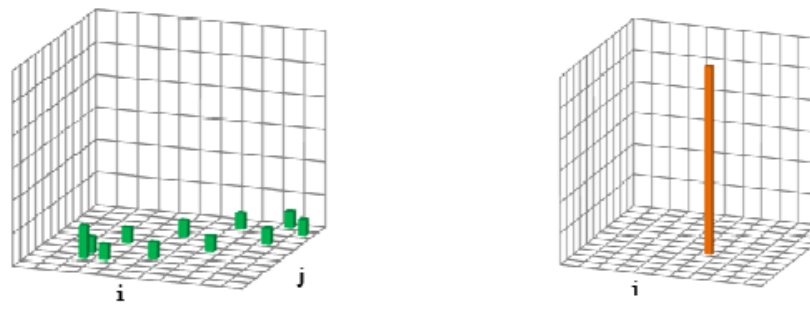

(b) time-domain co-occurrence matrices

Figure 3. Conceptual examples of time-domain cooccurrence matrix.

In our experiments, two kinds of pixel value were examined. The first one is surface reflectance. The second one is spectral cluster that is extracted by clustering in seven spectral bands for 46 scenes data set. It is expected that spectral clusters absorb undesirable fluctuation of surface reflectance. And time separation delta-t from one to six months were examined in order to search proper delta-t.

\subsection{Classifier}

The non-parametric minimum distance classifier was introduced for time-domain co-occurrence matrix. Euclidean distance $\mathrm{dE}(\mathrm{x}, \mathrm{c})$ and cosine distance $\operatorname{dn}(\mathrm{x}, \mathrm{c})$ between a pixel- $\mathrm{x}$ and $\mathrm{a}$ training class-c were examined in this experiments. The distance $\mathrm{dE}(\mathrm{x}, \mathrm{c})$ and $\mathrm{dn}(\mathrm{x}, \mathrm{c})$ are defined as Eq.(1) and Eq.(2), respectively.

$$
\begin{aligned}
& d_{E}(x, c)=\sum_{b=1}^{7} \sum_{i} \sum_{j}\left\{M_{x, b}(i, j)-M_{c, b}(i, j)\right\}^{2} \\
& d_{n}(x, c)=\frac{\sum_{b=1}^{7} \sum_{i} \sum_{j} M_{x, b}(i, j) M_{c, b}(i, j)}{\sqrt{\sum_{b=1}^{7} \sum_{i} \sum_{j} M^{2}{ }_{x, b}(i, j)} \sum_{b=1}^{7} \sum_{i} \sum_{j} M^{2} c, b(i, j)}
\end{aligned}
$$

$\mathrm{M}_{\mathrm{x}, \mathrm{b}}(\mathrm{i}, \mathrm{j})$ is a component (i,j) of the time-domain co-occurrence matrix measured from band-b in time-series data set for a pixel$\mathrm{x} . \mathrm{M}_{\mathrm{c}, \mathrm{b}}(\mathrm{i}, \mathrm{j})$ is that measured from band-b time-series data set for the training area of a class-c.

\section{CLASSIFICATION EXPERIMENTS}

\subsection{Land Cover Category}

Table 1 presents the land cover categories which are same with IGBP Land cover categories. These 17 categories were used in our classification experiments.

Table 1. Land cover categories(IGBP legend).

\begin{tabular}{ll}
\hline 1. Water & \\
2. Evergreen Needleleaf Forest \\
3. Evergreen Broadleaf Forest \\
4. Deciduous Need leaf Forest \\
5. Deciduous Broadleaf Forest \\
6. Mixed Forests \\
7. Closed Shrublands \\
8. Open Shrublands \\
9. Woody Savannas \\
10. Savannas \\
11. Grasslands \\
12. Permanent Wetlands \\
13. Croplands \\
14. Urban and built-up \\
15. Cropland/Natural Vegetation Mosaic \\
16. Permanent snow and ice \\
17. Barren/Sparsely vegetated
\end{tabular}

\subsection{Training and Accuracy Estimation}

84 classification classes were prepared for IGBP 17 categories, because each category consists of several classification classes. About 9,000 pixels on the average for each class and about 400,000 pixels in total have been extracted as training data. Figure 4 and Figure 5 show examples of training data for "evergreen needleleaf forest" and " barren/sparsely vegetated", respectively. Figure 6 shows examples of obtained time-domain co-occurrence matrix for "deciduous needleaf forest" and " savannas". 


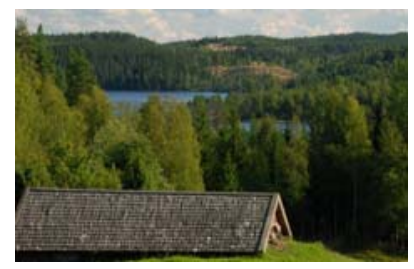

(a) ground view

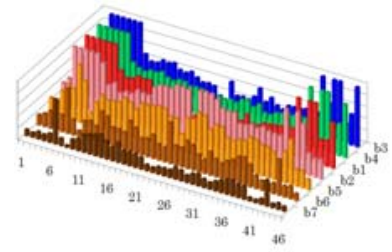

(c)time series reflectance of one pixel in the training area

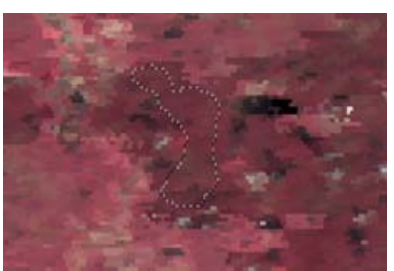

(b) training area

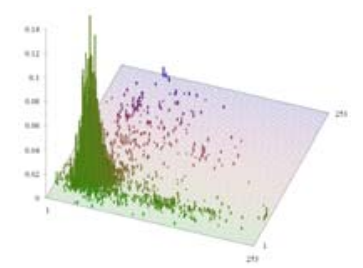

(d)time-domain co-occurrence matrix of the training area
Figure 4. Training data example for "evergreen needleleaf forest".

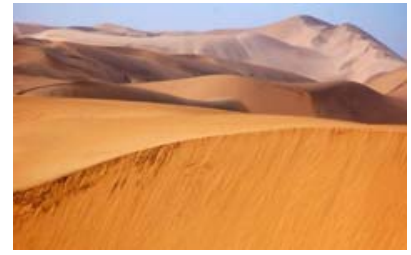

(a) ground view

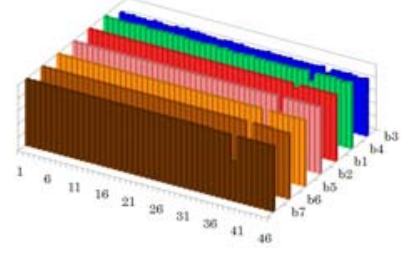

(c) time series reflectance of one pixel in the training area

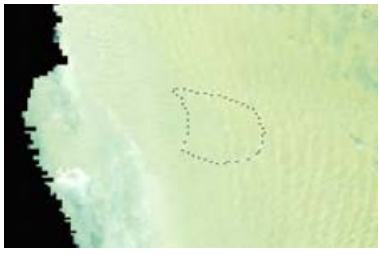

(b) training area

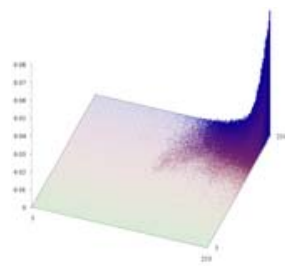

(d)time-domain co-occurrence matrix of the training area
Figure 5. Training data example for "barren/sparsely vegetated".

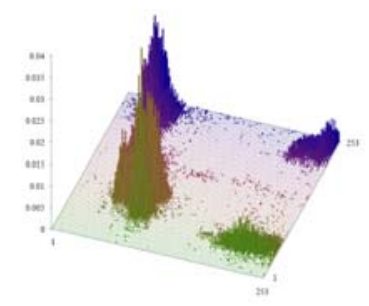

(a) "deciduous needleaf forest"

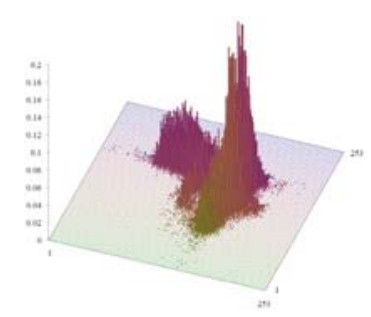

(b) " savannas"
Figure 6. Examples of obtained time-domain co-occurrence matrix.
Classification accuracies were measured by using test samples of 300 pixels that were sampled randomly from training area of each individual class.

\subsection{Classification Results}

Because the cosine distance classifier has always produced several percent higher mean producer's classification accuracy than that of the Euclidean distance classifier regardless of the time-separation delta-t, only the results when the cosine distance is used is shown in the following. When time-domain co-occurrence matrix is defined with reflectance, the highest accuracy of $95 \%-96 \%$ has been obtained for SR and NBAR products when time-separation delta-t is about 3 months as shown in Figure 7.

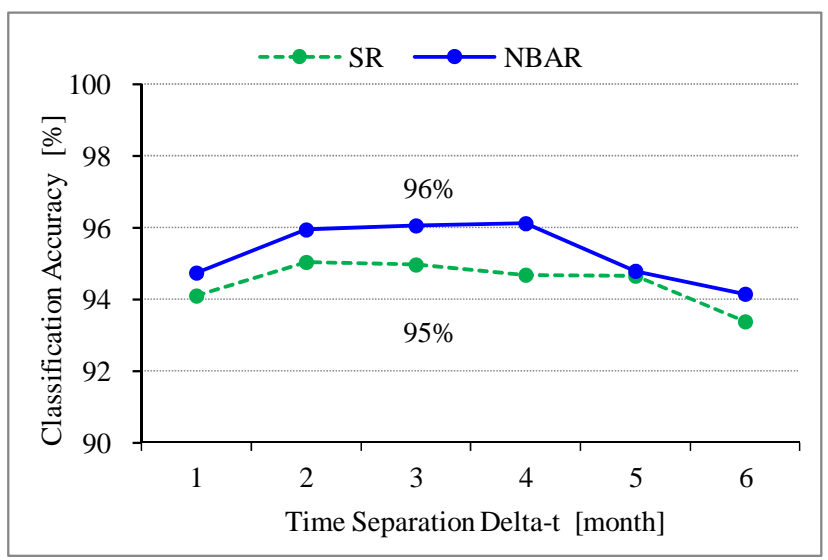

Figure 7. Classification accuracies obtained by time-domain co-occurrence matrix which is defined with reflectance.

When time domain co-occurrence matrix is defined with spectral cluster, classification accuracies are increased according to the number of clusters as shown in Figure 8. The highest accuracy of about $99 \%$ has been obtained for SR and NBAR products when time-separation delta-t is about 4 months as shown in Figure 9.

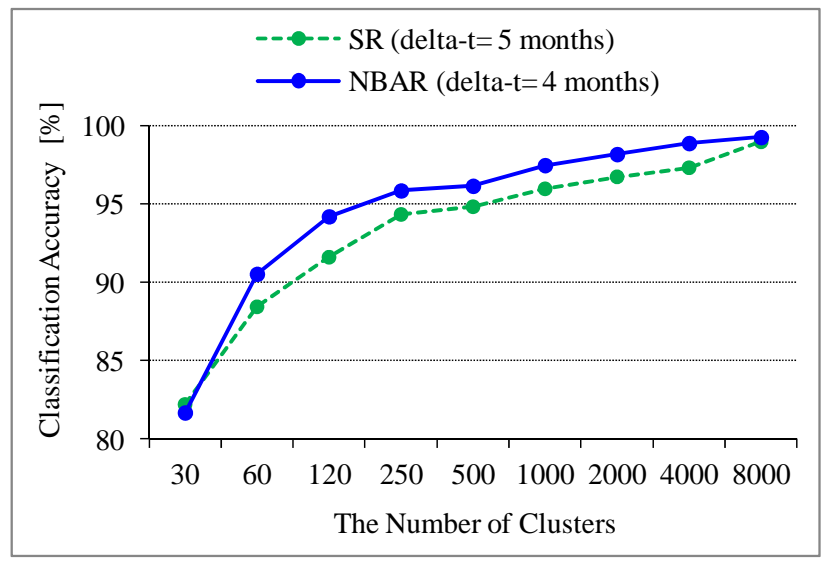

Figure 8. The relationship between the number of clusters and classification accuracies obtained by time-domain co-occurrence matrix which is defined with spectral cluster. 


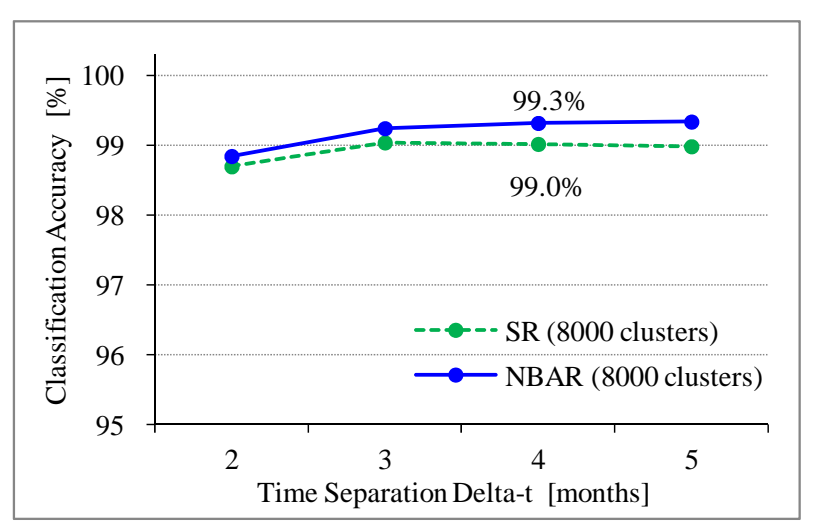

Figure 9. Classification accuracies obtained by time-domain co-occurrence matrix which is defined with 8000 spectral clusters.

The time-domain co-occurrence matrix defined with 8000 spectral cluster shows 3\%-4\% higher classification accuracy than that of the time-domain co-occurrence matrix defined with reflectance.

MOD12Q1 and MCD12Q1 of MODIS land cover product which are respectively produced from SR and NBAR products showed classification accuracy of $81 \%$ and $96 \%$, respectively. That is, the classification accuracy obtained by the time-domain co-occurrence matrix which is defined with spectral cluster is $3 \%-18 \%$ higher than that of MODIS land cover products. Classification accuracies of MODIS land cover product were measured by using same test samples. Because test samples were extracted from training area for classification of SR and NBAR products, it is fundamentally presumed that the accuracy of MOD12Q1 and MCD12Q1 products is lower than that of our classification results. However, we consider that these classification accuracies of SR and NBAR products showed good performance of the proposed simple classification method.

Figure 10 shows the classification results obtained by cosine distance classifier in the case of the highest accuracy.

\section{CONCLUSIONS}

Land cover classification for global scale were performed by using two kinds of multi-temporal MODIS reflectance products. The proposed method using the time-domain co-occurrence matrix and the non-parametric minimum distance classifier showed good classification performance compared with MOD12Q1 and MCD12Q1 MODIS land cover product.

The highest classification accuracy was obtained when the nonparametric cosine distance classifier was driven by the timedomain co-occurrence matrix defined with spectral cluster and three or four months time-separation. And also, it was cleared that Surface Reflectance 8-Day L3 product and Nadir BRDFAdjusted Reflectance product showed similar classification accuracy of $99 \%$ for IGBP-17 land cover categories.

Future study should be carry out in our classification scheme in order to examine stability of classification for multiple years and to validate classification accuracy with more suitable test samples.

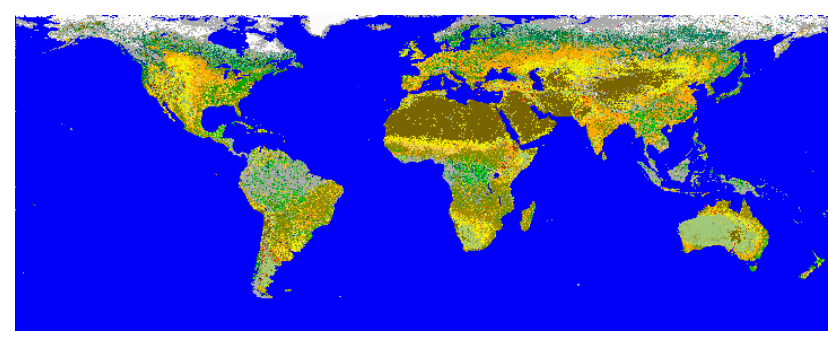

(a) SR product

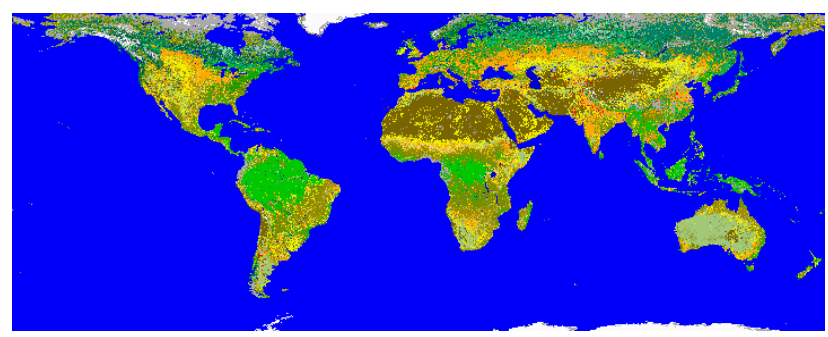

(b) NBAR product

Figure 10. Land cover classification results.

\section{Acknowledgements}

This study was supported by the JAXA GCOM-C project under contract "JX-PSPC-332027".

\section{REFERENCES}

Alan Srahler, Doug Muchoney, etc., 1 May 1999. MODIS Land Cover Product Algorithm Theoretical Basis Document (Version 5.0)

E. F. Vermote and S. Y. Kotchenova, March, 2008. MOD09 (Surface Reflectance) User's Guide. Land Processes DAAC(EROS) in collaboration with the Department of Mathematics and Computer Science, South Dakota School of Mines and Technology, January 2006. MODIS Reprojection Tool User's Manual(Release 3.3a).

Mikio Maeda, Kiyonari Fukue and Haruhisa Shimoda, 2010. Continental scale land cover classification using MODIS surface reflectance product. In: Proc. The 31th Asian Conference on Remote Sensing, Hanoi, Vietnam, pp.PS01-10-1PS01-10-6..

Haruhisa Shimoda and Kiyonari Fukue, 2011. CONTINENTAL SCALE LAND COVER CLASSIFICATION USING MODIS SURFACE REFLECTANCE PRODUCTS. In: 2011 IEEE International Geoscience \& Remote Sensing Symposiums, Vancouver, Canada, pp. 692-695.

Haruhisa Shimoda and Kiyonari Fukue, 2011. GLOBAL LAND COVER CLASSIFICATION USING MODIS SURFACE REFLECTANCE PRODUCTS. In: Proc. The 32nd Asian Conference on Remote Sensing, TAIPEI, pp.P_241_8-23-20-1-5. 